\title{
A case of periradicular surgery: apicoectomy and obturation of the apex, a bold act
}

\author{
Lino Lucio Locurcio', Rachel Leeson ${ }^{2}$ \\ ${ }^{I}$ Ashford \& St. Peter's Hospitals, Ashford TW15 3AA, UK. \\ ${ }^{2}$ Eastman Dental Hospital, London WC1X 8LD, UK.
}

Correspondence to: Dr. Lino Lucio Locurcio, Ashford \& St. Peter's Hospitals, London Road, Ashford TW15 3AA, UK. E-mail: lino.locurcio@gmail.com

How to cite this article: Locurcio LL, Leeson R. A case of periradicular surgery: apicoectomy and obturation of the apex, a bold act. Stomatological Dis Sci 2017;1:76-80.

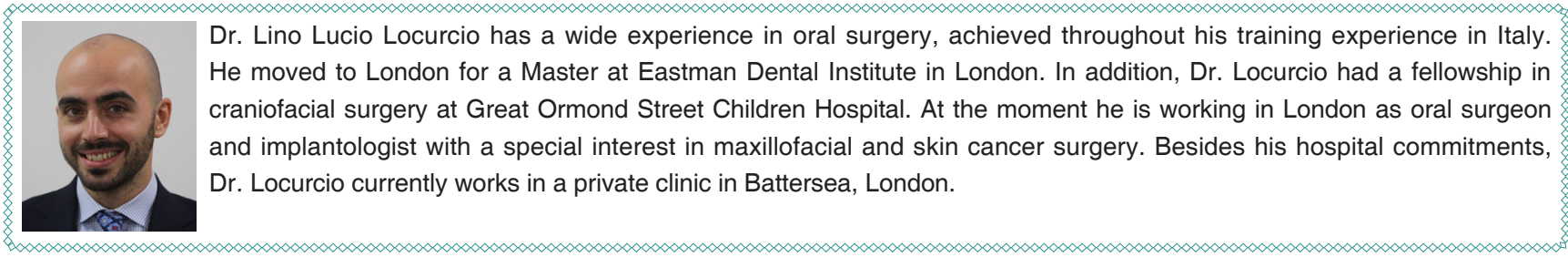

Article history:

Received: 08-10-2016

Accepted: 21-12-2016

Published: 29-06-2017

\section{Key words:}

Apicoectomy,

surgery,

flap,

root,

cyst

\section{ABSTRACT}

This paper reports a case of a recurrent periapical cyst treated with enucleation of the lesion, apicoectomy, and root end obturation on a lower left first molar. In the case of conventional root canal treatment failure, non-surgical retreatment is the preferred option in most of the cases. Several factors such as a complex root canal system or previous procedural accidents may impede the success of non-surgical retreatment. The authors have performed root-end resection and preparation under local anesthesia on a lower left first molar; The root canal filling is placed within the new cavity to close the path of communication between the infected root canal system and periradicular structures with an intermediate restorative material. The lesion was fully enucleated and sent for histopathology. The 29-year-old female patient was followed up at 2 weeks, 3 and 6 months presenting as a functional and symptomless tooth. Radiographic findings showed a clear and progressive refilling of the cavity with bone. All these factors highlight a positive prognosis for the tooth after periradicular surgery, which is now considered a valid treatment to keep the tooth as a functional unit in the oral cavity.

\section{INTRODUCTION}

An apicoectomy was well defined in 1884 by J. Farrar as "a bold act, which removes the entire cause [of disease] and which will lead to a permanent cure [...] may not be the best in the end, but the most humane." According to Black, ${ }^{[1]}$ the root-resection technique (amputation of the root apex) originated as a treatment for "pyorrhea alveolaris" complicated by a dental abscess in the late years of the 19th century as a valid alternative to a

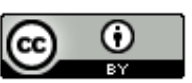

This is an open access article licensed under the terms of Creative Commons Attribution 4.0 International License (https://creativecommons.org/licenses/by/4.0/), which permits unrestricted use, distribution, and reproduction in any medium, as long as the original author is credited and the new creations are licensed under the identical terms.

For reprints contact: service@oaepublish.com

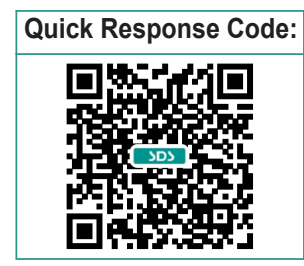


dental extraction. It seems that the first treatment properly described was performed on a 14-year-old boy in 1846, gaining access to the root with a trephine and a bur mounted on the dental engine to drill away the area of inflamed/infected tissue. ${ }^{[2]}$ After this, within a few years, a more radical method of complete excision of the root apex was performed and mastered. The pioneers in the apicoectomy technique started operating without any form of anesthesia and simply applied $95 \%$ phenol on the gingiva, then scraping away the inflamed tissue (dental abscess) until the alveolar bone covering the root end was exposed to the action of trephine and the straight bur. ${ }^{[1,2]}$ The first application of cocaine as a local anesthetic changed the reliability of this technique completely and of all other dental procedures. In 1907, procaine and novocaine were developed as a more stable substitute by A. Eihorn. Dr. T.P. Hinman of Atlanta (Georgia, USA), after examining the apicoectomy method, stated in a famous paper that "apicoectomy is rarely successful". This statement convinced the editor of Dental Items Journal to send letters of inquiry for opinions on this issue to several professors of wide reputation. ${ }^{[2]}$ Apparently, this controversy incited the clinicians to apply a new approach and to master a different perspective for the apicoectomy technique in which the ideal results were: (1) covering of the resected surface of the root by a new layer of cementum; (2) covering of the neoformed cementum by the periodontal membrane; and (3) space formerly occupied by the resected root should be filled by bone. In 1922, the first experimental study of root-resection was done by Bauler on cats in Germany. Bauler performed this procedure on six maxillary canines, and then after apicoectomy, histologic sections were obtained proving the growth of the periodontal membrane. ${ }^{[2]}$ Several other studies in the subsequent 30 years were conducted on animals and humans. Some of these studies described the possibility of filling the resected root with wax, lead or gold. ${ }^{[3]}$ Today, after previous endodontic failure, non-surgical revision of the root in cases of periapical infection, granuloma, or cyst is considered by many as the primary mode of case management. The decision to perform surgery is often open to deliberation and debate..$^{[1]}$ Apical surgery is often the last hope to save an endodontically treated tooth with a periapical lesion. The introduction of new materials for apical obturation in the early nineties improved the success rates of apical surgery to $80-90 \% .{ }^{[4]}$ As reported in the literature, the historical pathway to current surgical endodontic procedures and their applications has been tortuous and complex, heavily influenced by the European school. Many newer techniques, defined as "revolutionary", which are practiced today are simply a re-interpretation of surgical concepts that were lost in the archives of time. ${ }^{[5]}$ The advent of evidence-based endodontics has supported newer procedures with science and the usage of novel materials, modern techniques, and research outcomes. ${ }^{[5]}$

\section{CASE REPORT}

A 29-year-old female patient was referred by her dentist to our new patient's clinic (Oral Surgery Department, Eastman Dental Institute) for the extraction of the lower left first molar (LL6). Her referral letter clearly explained the previous attempts for preserving the tooth (LL6) with endodontic treatment and a cyst enucleation in the apex area under local anesthesia.

The low/mild anxious patient revealed her chief complaint as a "pulsating, throbbing pain" in the left mandible which she defined as intermittent and spontaneous, but was also evoked by percussion. Her second complaint was painful to touch intermittent swelling in the buccal aspect immediately below the gingival sulcus of the LL6. She reported that her dentist in Spain tried to restore the tooth with endodontic treatment and also performed an operation with local anesthesia. A cyst was removed related to the apices of LL6 as included in the referral letter. The dentist's attempts to preserve the tooth had limited success because she kept feeling pain. A periapical X-ray demonstrated persistent bone resorption. She had recurrent infections which needed to be treated with antibiotics. No sensory loss or numb/tingling sensation had ever been reported by the patient. Medical history: the patient is fit and well; the patient denied any major systemic disease. Social history: moderate/social consumption of alcohol; smoker: 10-15 cigarettes per day. Low/mild dental anxiety levels as reported by the pre-assessment questionnaire, but the patient was amendable to local anesthesia and already has undergone prolonged dental procedures under local anesthesia. In an attachment to the dentist's letter was a small periapical X-ray [Figure 1] of the LL6. Due to routine procedures and the poor visualization of the periapical X-ray, an orthopantomogram X-ray [Figure 2] was requested on the same day and examined. Considering the case, the surgeon and the clinic's supervisor suggested to the patient to undergo an apicoectomy to preserve the tooth potentially. The $X$-ray examination showed a composite filling and a well-done root canal filling on the LL6 with a periapical radiolucency $1 \mathrm{~cm}$ circa (reported by the dentist as a recurrent cyst). The cyst is in intimate contact with the inferior alveolar nerve canal [Figures 1 and 2].

On the day of the surgery, the patient was admitted to the clinic, the surgical procedures were described, the 


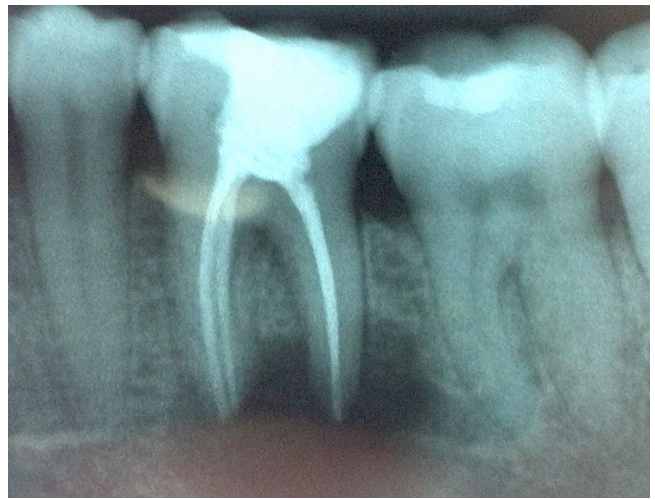

Figure 1: Periapical view X-ray of the lower left first molar apex

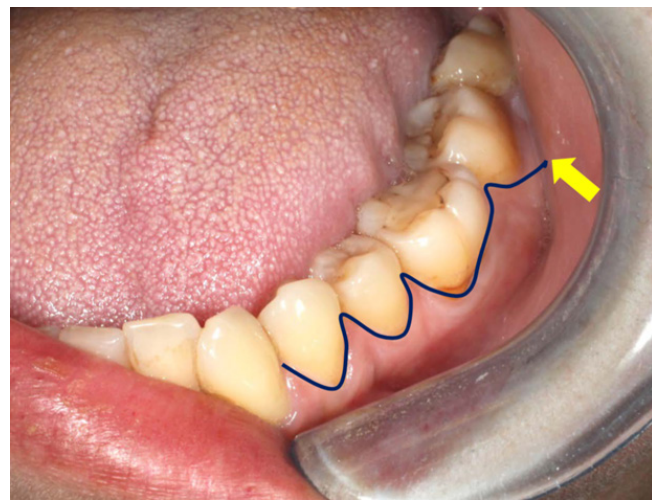

Figure 3: Open-angled distal relieving incision mucoperiosteal flap, based on a variant design of Dr. Lino L. Locurcio

risks and benefits were explained, and a consent form was signed. The patient sat comfortably on the chair and asked to complete one preoperative mouthrinse with $0.15 \%$ chlorhexidine. The sterile surgical tray was opened, sterile drapes and protective glasses were used to cover the patient. Local anesthesia was performed using 3 cartridges of Lignospan ${ }^{\circledR}(2 \%$ lidocaine with 1:80,000 epinephrine injection solution $2.2 \mathrm{~mL} /$ cartridge); 1 cartridge for the lower left inferior alveolar block plus 2 cartridges used for wide buccal infiltration from lower left 4 to 8 .

\section{Flap design}

According to the classic literature, the most commonly used mucoperiosteal flaps for apical access in the posterior part of the mandible are the envelope flap and the triangular flap with an obvious mesial releasing incision. The releasing incision preserves the distal vascular branches from the buccal artery. Considering the proximity to the mental foramen, we elected to choose a different surgical approach that allowed us to gain better access to the LL6 root. This surgical approach increases visibility, reduces the amount of exposed bone, and reduces the risk of mental nerve damage. The supervisor and the first operating surgeon proposed this mucoperiosteal flap with a wide

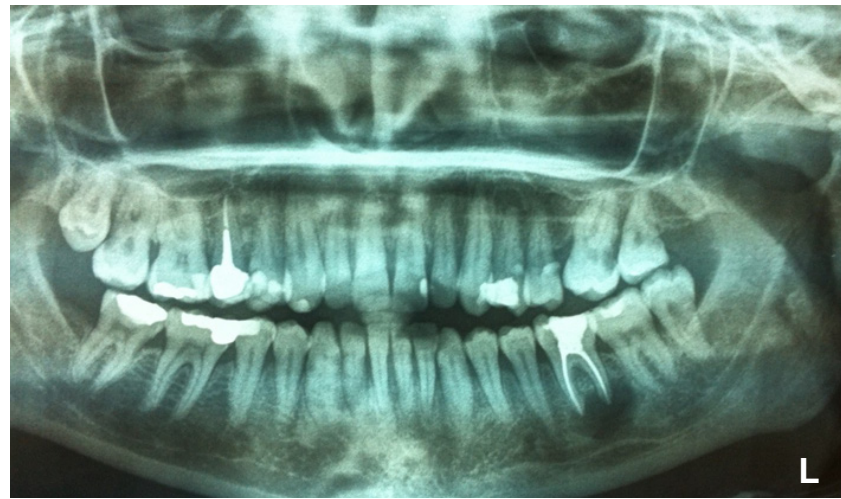

Figure 2: Orthopantomogram X-ray

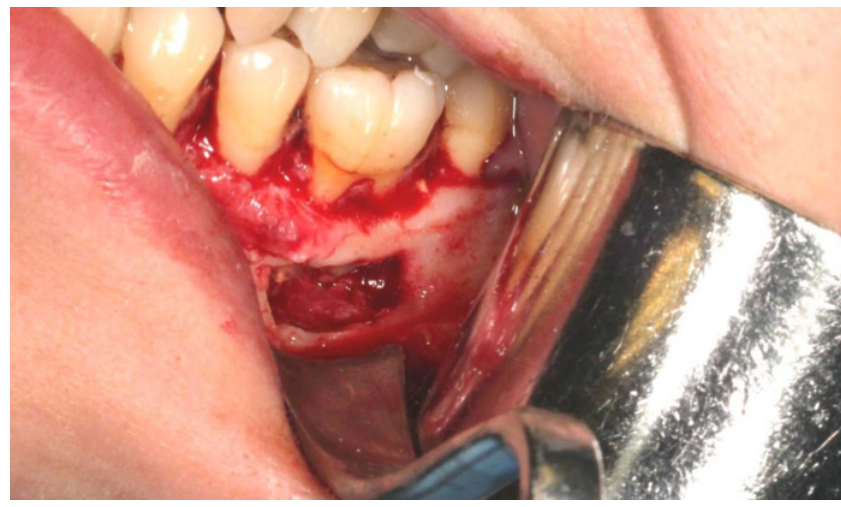

Figure 4: Cavity access-in orange: mesial root gutta percha filling

angled distal relieving incision [Figure 3].

\section{Visualization and ostectomy}

After elevating the flap and visualizing the cortical bone, immediately a continuous soft tissue structure was noted between the bony cavity and the buccal flap. After dissection, the buccal bone showed a hollow space slightly distal to the LL6. Ostectomy was performed successfully with a straight fissure carbide bur with the opportunity to enlarge the bony defect to a buccal window $(1 \mathrm{~cm}$ circa) and gaining access to the cystic structure and the LL6 roots [Figure 4].

The process of removal of the cystic lesion was incomplete in the lower part of the cavity. In fact, because of the proximity of the alveolar nerve, curettage provoked an intense "stabbing" sensation while attempting to elevate the bottom of the cystic surrounding fibrous tissue. This discomfort led the surgeon to choose a more conservative approach during the operation. Subsequent histopathological examination of the samples reported the lesion as a benign recurrent radicular cyst.

Astraight fissure carbide bur was directed perpendicular to the long axis of each root and used with full rotating power to cut the distal $3 \mathrm{~mm}$ of the apex. This cut 


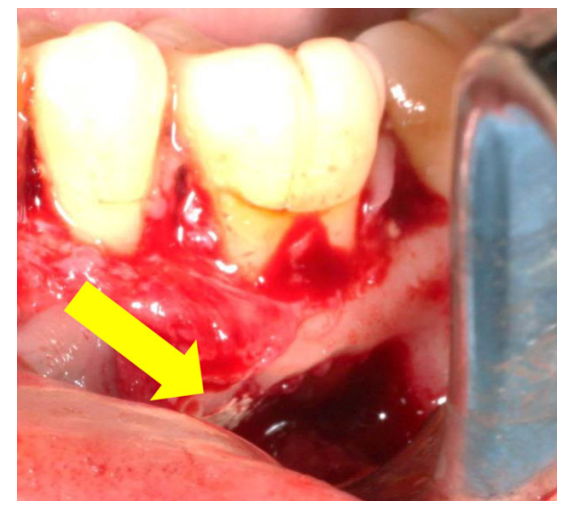

Figure 5: Obturation phase-partial cortical bone visible; yellow arrow points to IRM

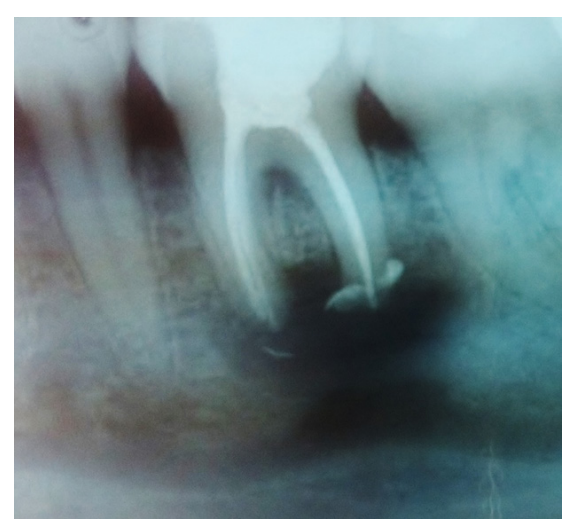

Figure 6: Postoperative periapical X-ray of the lower left first molar

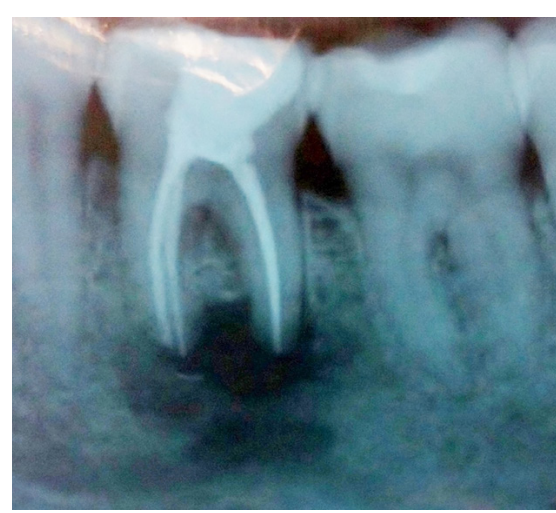

Figure 7: Six months post-operative periapical X-ray of the lower left first molar; interim restorative material barely visible

is completed in order to decrease dentin tubules peripheral microfiltration. This cut exposed the gutta percha material and removed any excess gutta percha protruding from the apical foramen. Saline irrigation was used to clean the cavity, removing debris of guttapercha, dentin, and cementum (30 s). Sterile gauzes in long strips were gently placed inside the empty cavity to obtain hemostasis, to clean the environment. The strips were removed after $2 \mathrm{~min}$. The 3 apices of the roots were visualized and then carefully resected at $90^{\circ}$ with a straight fissure bur on a hand-piece. After retrograde preparation with manual instruments, the canals were obturated with interim restorative material (IRM) (oxide-zinc eugenol cement). After proper preparation of the IRM's components, the IRM was carried onto a microspatula and placed into the newly formed cavities in each apex (respectively starting from mesiolingual to mesiobuccal and distal roots apex) [Figure 5]. Any excess of IRM was removed with a small rounded bur with no saline irrigation. The careful use of a manual bone trimmer allowed us to plane and remove any blunt border from the window access. The trimming was completed to enhance soft tissue juxtaposition and healing. Before closing the flap, the removal of periradicular fibrous tissue was performed with the hope of gaining a thinner, less prone to inflammation gingiva, even if the clinical outcome would have shown a slight recession of the gingival margin. The area has no esthetic relevance, so the patient was satisfied.

\section{DISCUSSION}

After the failure of the conventional root canal treatment (RCT), non-surgical retreatment is the preferred option in most cases. Several factors, such as a complex root canal system or previous procedural accidents, may impede the success of non-surgical retreatment. In these cases, periradicular surgery and apicoectomy would be the treatment of choice to preserve the tooth.

Performed root-end resection and preparation, the root canal filling is placed within the created cavity to close the path of communication between infected root canal system and periradicular tissues. Using a root canal filling material with ideal properties has an immense effect on the treatment outcome of the surgery. An ideal material should be non-absorbable, non-corrosive, noncytotoxic, not affected by moisture, dimensionally stable, biocompatible, antibacterial, radiopaque, cost-effective, easily manipulated, adhesive to dentinal walls, create a tight seal, and induce cementogenesis. ${ }^{[6]}$

Numerous materials have been recommended for rootend obturation, and many studies have attempted to identify an ideal material; however, an ideal material has not been found. ${ }^{[7]}$ The most commonly used materials are mineral trioxide aggregate (MTA) and IRM. ${ }^{[7]}$ Recent studies show that although IRM achieved the most favorable healing response, both radiographically and histologically, these results were not statistically different from MTA. ${ }^{[8,9]}$ Our school regularly uses IRM as an apical surgery retrograde filling.

The follow-up examinations of this case were after 2 weeks, 3 months and 6 months. The examinations noted an absence of symptoms, such as pain, 
swelling, trismus and the normal function of the tooth was preserved. No sensory loss was reported by the patient. X-rays taken immediately postoperatively showed the IRM in place [Figure 6]. The X-ray after 6 months showed a reduction of the cavity and the initial appearance of a thin layer of bone separating the inferior alveolar canal from the surrounding cortical alveolar bone [Figure 7].

The patient is happy and satisfied to have her tooth functional. Also, the X-ray evaluation reported clear and slow bone deposition in the cavity after apicoectomy, obturation, and cystic enucleation. These criteria highlight the success of the treatment after 6 months. Therefore, after the previous failure of the endodontic treatment, we can consider this a valid treatment.

This is a bold act, with a high rate of success that must be considered on certain occasions by the experienced surgeon. This procedure was suggested to the patient as an act of pure preservation and to respect the anatomo-functional importance of the tooth. ${ }^{[10]}$

\section{Authors' contributions}

Manuscript's preparation: L.L. Locurcio, R. Leeson

Concept design: L.L. Locurcio, R. Leeson

Literature search: L.L. Locurcio

Manuscript's review: L.L. Locurcio

\section{Acknowledgments}

We thank Dr. Jon Wagner for his contribution in language editing.

\section{Financial support and sponsorship}

None.

\section{Conflicts of interest}

There are no conflicts of interest.

\section{Patient consent}

Obtained.

\section{Ethics approval}

All the procedures followed were in accordance with the ethical standards of the responsible committee on human experimentation (institutional or regional) and with the Helsinki Declaration when reporting studies on human beings.

\section{REFERENCES}

1. Black CV. Amputation of the roots of teeth. In: Litch WF, editor. The American system of dentistry. Philadelphia: Lea Brothers; 1886. p. 990-2.

2. Ojha GD. The historical changes in the tooth and surrounding structure following apicoectomy in dogs. [dissertation]. Chicago, IL: Loyola University; 1961.

3. Gutmann JL, Gutmann MS. Historical perspectives on the evolution of surgical procedures in endodontics. $J$ Hist Dent 2010;58:1-42.

4. von Arx T, Peñarrocha M, Jensen S. Prognostic factors in apical surgery with root-end filling: a meta-analysis. J Endod 2010;36:957-73.

5. Gutmann JL, Gutmann MS. Historical perspectives on the evolution of surgical procedures in endodontics. $J$ Hist Dent 2010;58:1-42.

6. Asgary S, Ehsani S. Periradicular surgery of human permanent teeth with calcium-enriched mixture cement. Iran Endod J 2013;8:140-4.

7. Tawil PZ, Trope M, Curran AE, Caplan DJ, Kirakozova A, Duggan DJ, Teixeira FB. Periapical microsurgery: an in vivo evaluation of endodontic root-end filling materials. $J$ Endod 2009;35:357-62.

8. Lindeboom JA, Frenken JW, Kroon FH, van den Akker HP. A comparative prospective randomized clinical study of MTA and IRM as root-end filling materials in single-rooted teeth in endodontic surgery. Oral Surg Oral Med Oral Pathol Oral Radiol Endod 2005;100:495-500.

9. Chong BS, Pitt Ford TR, Hudson MB. A prospective clinical study of mineral trioxide aggregate and IRM when used as root-end filling materials in endodontic surgery. Int Endod J 2009;36:520-6.

10. Ioannides $\mathrm{C}$, Borstlap WA. Apicoectomy on molars: a clinical and radiographical study. Int J Oral Surg 1983;12:73-9. 